\title{
Objects, Metrics and Practices: An Inquiry into the Programmatic Advertising Ecosystem
}

\author{
Cristina Alaimo ${ }^{1}$ and Jannis Kallinikos ${ }^{2}$ \\ ${ }^{1}$ Surrey Business School, University of Surrey, Guildford, UK \\ ${ }^{2}$ LSE, London School of Economics, London, UK
}

\begin{abstract}
Programmatic advertising is a large scale, real-time bidding process, whereby ads are automatically assigned to available spaces across types of media and geographic regions upon an individual user's browser request. The large-scale automation of programmatic advertising requires the establishment of standards and the development of technologies to govern the behavior of market participants (sellers, buyers, intermediaries). We present evidence on the rules of programmatic exchange and on the role played by a specific class of digital objects, focusing in particular on the metrics to which they are linked and how they define both the objects exchanged and the parameters of these exchanges. We furthermore demonstrate that the metrics and the technological complexes associated with them are constituted by the institutional field of digital advertising and its complex technological infrastructure. Rather than being simply means to monitor a pre-existing reality 'out there' (such as user or audience behavior) these metrics and techniques bring forward their own reality and heavily impact upon and shape the objects and processes of digital advertising.
\end{abstract}

Keywords: Automation, Digital Advertising, Performativity, Digital Objects, Information Infrastructures.

\section{Introduction}

Over the last two decades, the field of advertising has been undergoing dramatic transformations that are reshaping the very process of advertising and remaking the fundamental objects and actors involved in that process. This is forcefully evidenced by the recent diffusion of what is referred to as programmatic advertising, whereby ads are displayed to viewers or readers through a real time auction in a process of dizzying computational and organizational complexity. We view advertising as a social field or practice and we aim, accordingly, to document shifts in that practice that result from the introduction of new objects and metrics, exchanges between actors and systems, and, ultimately, economic and power relations. The intersection of economic pursuits with technology and their links to online media make the field of programmatic advertising a particularly germane object of study that links to central concerns of the conference call. 
Advertisement -the process of calling user's attention to a product or service by way of paid announcements- has always been contingent on the assignment of advert content to individuals. This process has traditionally occurred via the construct of the audience, an anonymous and rather sizeable cohort of readers, viewers or listeners that are supposed to share a few fundamental attributes to which an advert seeks to appeal $[29,31]$. The debate on the facticity of audiences has a long history in sociology and media studies [see i.e. 4, 5, 6, 30]. Ettema and Whitney speak of "institutionally effective audiences" [13, p. 5], a concept recently expanded by Napoli to signify audiences that are "constructed and defined to reflect the economic and strategic imperatives of media organizations" and that "can be effectively integrated into the economics of media industries" [24, p. 3]. Yet, IS scholarship is surprisingly lacking in debating the facticity of concepts such as "user attention" or "audiences", a fact that becomes regretful in light of the recent technological advancements and the proliferation of metrics and tracking devices that are linked to the automation of advertising exchanges. The knowledge gap on the functioning of digital media buying and exchange and their connected technologies is even more striking if one considers the history and evolution of digital advertising and its profound interdependence with the history of the web that attests to the importance advertising-based business models have had for the majority of content, information and entertainment providers.

In what follows we present preliminary evidence from an ongoing study of the process through which programmatic advertising operates. To grasp and better appreciate the current transformations we retrace the recent evolution of the digital advertising industry and its leaning toward user measurement and profiling. The reliance of publishers to advertising-funded business models and the skepticism with which marketers originally met the new medium fueled a rush to develop a range of data tracking devices, better tools for measuring, and the consequent mushrooming of data analytics companies which help publishers find convincing ways to demonstrate marketers' return on investments (ROIs). Recently, the plunge toward more effective ways of buying and selling ads online led to the rise of programmatic advertising and the implementation of massive centralized virtual marketplaces called ad exchanges that offer in real-time individual user profiles to bid on (Google owns one of the biggest ad exchange platforms). Programmatic advertising is rapidly diffusing as a faster, cheaper, and scalable alternative to previous methods of ad exchanges such as ad networks and direct exchanges.

In its essence, programmatic advertising is a huge, real-time bidding process, whereby ads are automatically assigned to media spaces across types of media and geographic regions upon an individual user's browser request. This means that the entire ecosystem's exchange with its hundreds of platforms operates "on-demand", every time a user's browser opens a publisher website and triggers a real-time request for an ad. The whole exchange is usually completed under 100 milli-seconds and remains entirely invisible to the user who may experience a small lag in loading the publisher page. Dubbed as the "holy grail of targeting" [see 29, p. 79], the automated and real-time nature of ad exchanges characteristic of programmatic advertising requires the establishment of standards and the development of technologies to govern the behavior of market participants (sellers, buyers, intermediaries). Requests for real 
time bidding on available ad slots have to enter the ecosystem in appropriate data formats and be exchanged using data objects, standardized conventions and IT-based solutions that allow real-time computation to occur in an unprecedented, almost dizzying, scale.

The diffusion of automated exchanges and the move toward selling user attention on demand opens yet another chapter in the evolution of digital advertising and, consequently, of the web. Key in the developments we point to is the diffusion of complex and distributed automated marketplaces which is itself contingent on the introduction of a particular class of digital objects. In what follows, we recount their operations and the measures they black-box. We frame our empirical study against the background of tracking technologies linked to online marketing and we further draw on theories of information infrastructures [7, 10, 16, 33] and other contributions across the social sciences $[11,12,21,22]$ to reflect and theorize on our empirical findings. The evidence we present indicates that the specific class of digital objects that underlie programmatic advertising is closely linked to several metrics that define both the objects exchanged and the parameters of these exchanges. We show the path dependence of such metrics and demonstrate that the metrics and the technological complexes associated with them are constituted by the institutional matrix of digital advertising and its complex technological infrastructure. Rather than being simply means to monitor a pre-existing reality 'out there' (such as user or audience behavior) these metrics and techniques bring forward their own reality and heavily impact upon and shape the objects of digital advertising.

This paper is organized as follows: in the next section we trace the history of digital advertising through the evolution of its digital objects. After having presented our research design, we describe the findings of our empirical study focusing on the protocol, measures and digital objects that constitute the programmatic exchange. Following it, we discuss and reflect on the facticity of these data objects and link our reflections to broader concerns on the performative role they have at the industry level.

\section{The Evolution of the Field of Digital Advertising}

Since the beginning of the web, online publishers (the majority of content producers such as newspapers and blogs and later also content distributors such as social media) understood that a paid subscription model was not a tenable business option and decided to rely on advertising-based business models. From that point on (early 1990s), the development of the web content has been inherently linked to the development of the digital advertising industry, its dynamics and technological evolution. Publishers found themselves competing in an ever-increasing fragmented space to convince marketers of the effectiveness of online advertising spending on their own websites [see 29]. This in turn, quickly brought the mushrooming of data tracking devices, measuring tools and data analytics companies.

In the mid-1990s, in fact, a systematic measurement of internet audiences did not exist. The newly born digital advertising industry rushed to assemble a toolkit of 
standards, measures and a lexicon borrowed from traditional commercial media and adjusted to the web. Some of the old measures and standards persisted such as the case, for instance, with the one used for the price setting. CPM or cost per mil (thousand) has been the traditional standard price model for newspapers and other mass media. In the CPM model, the price of a single ad is determined by the cost of reaching one thousand individuals. The newly instituted sector maintained also and relied on the concept of the ad impression used by traditional commercial media. The concept has always been contested, yet its adoption online added more ambiguity with respect to what constitutes an online impression. The basic definition of [an] "advertisement that was sent to an individual who had clicked on a site's page" [29] does not specify if the ad is delivered on the site's page or to what extent an individual may effectively view the ad. As we show further ahead in the empirical section, these are fundamental aspects upon which the measurability of programmatic exchange and the cost of what is exchanged are constantly negotiated.

Other traditional methods of audience measurement crumbled under the changes imposed by the new digital medium. The fragmentation of offering in the online medium and the consequent dispersion of audiences across a number of sites rendered questionable the relevance of traditional panel-based models of audience measurement (like the Nielsen model) [see 6, 24, 25]. The difficulties in measuring audiences and the skepticism of marketers and big clients toward the digital medium pushed publishers and tech entrepreneurs to finding new ways to assess the presence of users and their activities online. One such way was indeed to track the user "click". Clicks not only gave advertiser evidence of the presence of users but it produced as well the detailed trace of their online activities. Publishers were quick in understanding that the measurability of "clicks" was one of the absolute novelties of the new medium. For the first time, marketers could have evidence of the effectiveness of their spending in advertising campaigns. It comes as no surprise that since roughly 1993 until today the digital advertising industry has grown spiraling around the central assumption that success in the business was a matter of finding more and more accurate ways of measuring audience and to eventually prove ROIs. Napoli defines the new concept of "audience" brought about by the proliferation of digital measuring and tracking technologies as "audience information systems" [24]. The term aptly signifies the major shift in the conception of audience caused by the use of data analytics $[1,2,5$, $6,28]$. To us, it also clearly points to the broader changes such shifts caused in the dynamics and organization of the digital advertising domain.

The complex relationship between publishers (or sellers) and marketers (or buyers) essentially developed on issues of measurability up to such extent that the entire evolution of the sector can be narrated in terms of measures and its associated devices. The evolution of the digital advertising industry has been driven by (i) crucial technological developments such as the development of the web browser, tracking technologies and automation; (ii) existing advertising industry dynamics, such as the concentration and increasing power of media-buying and -planning and their consequent push to audience measurability; and (iii) the overreliance of web content producers (from big publishers to individual blogs) on versions of an advertising-business model. Within this paradigm, crucial technological developments coinciding with the 
implementation of a class of digital objects such as the cookies, the web beacons, the pixel and other tracking and measuring devices, together with what they measure and how, stands out as key to understanding the evolution of the field of digital advertising toward the rationalization and measurement of audiences and the influence it exerts on the evolution of the web.

Galloway aptly defines the web browser as a "hiding machine," given that its main filtering and translating function actually hides the web [14]. The browser uses a set of instructions (HTML) to filter, organize and display content. The browser allows hyperlinking and marks a difference with the preceding text-only based web. For the digital advertising industry, the browser allows the development of more sophisticated forms of graphic advertising, linking and the hiding of tracking devices. Cookies were invented by Lou Montulli in 1994 working for Netscape Communication to solve a shopping problem $[9,29]$. In the original HTTP protocol, each request made by a user's computer to a web browser would be treated as new. Shopping carts back then had no way of linking different items to the same user. Machines read different items as different choices of different users. To solve this issue Montulli created a small text file that a website could place in a user computer with an identification code for the user and its activities (clicks), effectively solving the problem of user identification within and across sessions. Something invented as a solution to a relatively small problem led to the development of an entire industry of tracking which still constitutes the backbone of web advertising. Cookies gave computers a memory and user browsers the authorization of storing user histories by design without consent or knowledge from users [9]. The use of cookies quickly proliferated. Netscape installed cookie-capabilities into Navigator in 1994, followed by Microsoft's Internet Explorer in 1995.

The reaction of the industry was immediate and brought the development of a set of related industry practices and new service-related companies. The fact that cookies were implemented with a "same origin policy" - meaning that only the originator of the cookie could read the information stored in it, led to the emergence of ad networks. These alliances of publisher websites often led by an intermediary emerged to implement cross-sites tracking of users (with the so called "third-party" cookies) and, in so doing, they offered better audience analytics and measurement to advertisers $[26,29]$.

The activity of ad networks became fundamental to the field of digital advertising for a number of reasons. Ad networks offered a solution to the problem caused by the increasing fragmentation of content -what has been called the "long tail" [3] and the fact that audiences, differently from traditional commercial media, were spread across a number of different small websites. Ad networks aggregated publishers (called affiliates) and exchanged the possibility of installing and reading cookies across websites for a share of the revenue they made by selling the possibility to reach a certain kind of audience. They grew from accounting for 5\% of ad impressions sold in 2006 to $30 \%$ of ad impressions sold in 2007 [IAB in 24, p. 71]. One of the reasons of this success was that ad networks capitalized on the oversupply of publisher inventory; that is, the available ad slots in a publisher's online space. This remnant inventory was thus aggregated and auctioned by ad networks to media buyers at a discounted 
price. Although ad networks helped publishers sell their remnant inventory (which on average accounted for the $80 \%$ of the total inventory) the long-term effect of their operations was to move the attention of media buyers and planners from contexts (i.e. the publisher site or its reputation) to users [29]. The ad network auction system let marketers bid for impressions but did not reveal the websites where the impression was being delivered. Effectively what was on offer was the trackability of users rather than an ad space on a reputable publisher outlet.

One of the most important ad networks was DoubleClick. Founded in 1995, the company has been at the center of the media attention several times, most notably for having merged with Abacus Direct, an offline database of consumers amidst fear of privacy breach, for having been investigated by The Federal Trade Commission and for having been bought by Google in 2007. DoubleClick also implemented the first automated version of ad serving technology with its DART (Dynamic Advertising Reporting \& Targeting) a software which automated the ad buying process for advertisers and minimized unsold inventory for publishers. Ad serving technology is an important building block of today's media buying and planning. The basic functionalities of ad serving technology gradually evolved to respond to the need of publishers that wanted to sell the same ad slot to more than one marketer. At the beginning, ad serving was made of pieces of codes that rotated different creatives (the content of an ad) into a single ad slot on a publisher's site. This was followed by the development of a set of metrics to optimize the ad delivery. A ranking system called waterfall rotated the different creatives on the basis of a number of pre-set goals and performance metrics that related to user click, post-click and post-impression activities and interactions. The data from these basic functionalities were gradually incorporated into more sophisticated versions of serving technology like the DART system that was able to support decision making in an increasingly automated way. The system was eventually integrated with Google DoubleClick ad exchange, the centralized marketplace of programmatic advertising ecosystem.

\section{$3 \quad$ Research Design and Methodology}

The research is based on case study research design that combines the investigation of the field of programmatic advertising (the case) with a field-embedded case study of Smartology an independent, demand-side platform and trading desk [32].

The field-embedded study has been conducted over a period of 24 months in the company headquarters in London, UK and entailed two periods of 5 and 7 months respectively, with a year of follow-up communications and industry research in between. The first period covered the work of the company in the direct (traditional) digital advertising ecosystem. The second extended into the migration of the company to the programmatic advertising and its integration with the most important ad exchange platforms: Google Ad Exchange and Index Ad Exchange. Data were collected through semi structured ethnographic interviews (20), direct observations, company's internal documents (50) including reports, system screenshots, console screenshots, live logs examples and demonstrations. Additional data on the field of programmatic 
advertising have been collected with industry expert interviews (Google AdSense, Pangaea Alliance, Xaxis) but also a large range of industry documents, including Interactive Advertising Bureau (IAB) publicly available documents (35), other external documents (55), US patent applications (12) and other secondary resources such as industry reports, news articles and whitepapers.

Data were collected and analyzed following Yin's [32] procedure of constructing a case study database and case study protocol to maintain a coherent narrative and chain of events. Shifting between the two levels (filed and company) has been essential to acquiring a more complete and nuance view of the complexity of programmatic advertising.

\section{The Programmatic Ecosystem: Facts and Processes}

The drive toward effective ways of buying and selling ads online has led to the establishment of massive centralized virtual marketplaces called ad exchanges. Such marketplaces essentially overcome the fragmentation of online media and advertisers through massive, real time auction processes that evolve around the browsing behaviors (ad impressions) of huge user crowds. These centralized and automated buying systems hosted by ad exchange are rapidly diffusing as a more efficient alternative to the methods of ad exchanges reviewed in the previous section hosted by ad networks. In what follows, we paint the work of ad exchanges in large brush strokes.

The automated method of buying and selling ads in large scale, distributed ecosystems happens between platforms that act on behalf of traditional actors such as online publishers and marketers (buyers, sellers) and novel actors such as various data brokers and intermediaries. Demand Side Platforms (DSP that act on behalf of marketers), Supply Side Platforms (SSP that act on behalf of publishers) and many Data Management Platforms (DMP) exchange massive flows of data in real time as they seek to buy individual user attention. As indicated, the entire ecosystem's exchange, with its hundreds of platforms, operates "on-demand", every time a user's browser opens a publisher's website and triggers a real-time request for an ad. The massive, automated and real-time nature of ad exchanges occurring in the programmatic advertising ecosystem requires the establishment of standards and technologies to govern the behavior of market participants (DSP, SSP and SMP) and deal successfully with the protean data tasks their exchanges requires.

The Real-Time Bidding (RTB) protocol is the rule-book of the auctioning process in the field. The RTB Protocol derives from a concerted work undertaken by a group of demand-side and supply-side platforms in 2010. Under the supervision of the IAB, these platforms started to lay down the standards for the programmatic exchange. Following the adoption success, OpenRTB was introduced as an IAB standard in January 2012. JSON (JavaScript Object Notation) is the suggested format for bid request and bid response data payloads but not the only one adopted. Google AdX, for instance, offers three protocol options; (1) Ad Exchange protobuf, (2) Open RTB protobuf and (3) Open RTB JSON. Meanwhile, Index Exchange, an independent Toronto-based ad exchange, only supports Open RTB JSON. 
Fig. 1 illustrates the Open RTB exchange ecosystem, although it depicts the flow as unidirectional exchanges, most of what happens in the ecosystem is interactive and runs in both directions.

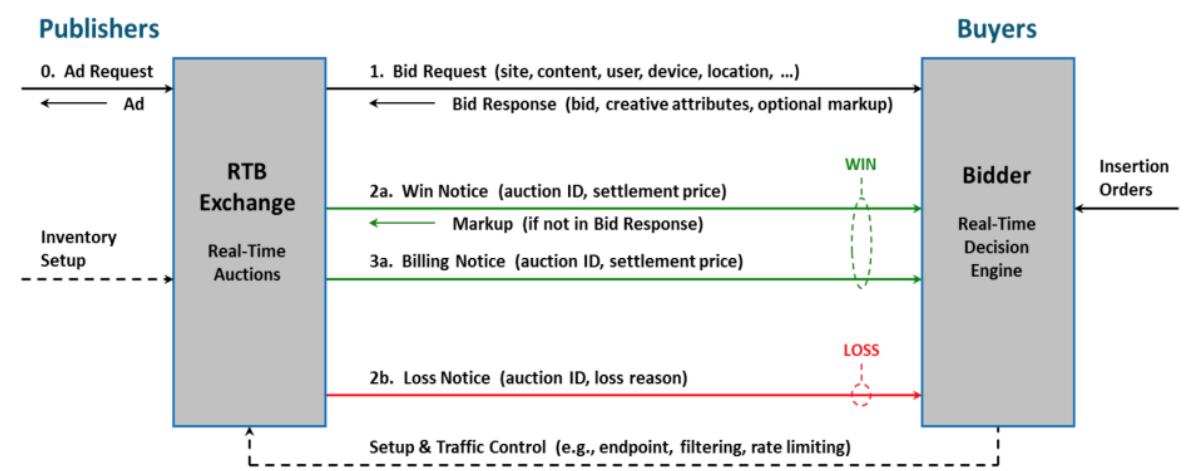

Fig. 1. Schematic representation of RTB exchange [18]. [From the top left] 0. A user browses online and opens a newspaper article (or any other content-based website). In so doing, the user's browser produces an ad impression. The article's ad inventory has been listed by the newspaper in a Supply Side Platform (SSP) (for instance, DoubleClick for publishers). 1. The ad exchange platform issues a "bid request" for the given impression of an ad slot. The different "bidders" or Demand Side Platforms (DSP) connected to the Google ad exchange platform will receive a "bid request" object, with details of the impression being sold, sometimes including minimum CPM (cost per mille impression), and other attributes or parameters. The DSP bidders respond with a "bid response" object, if the bid request they receive is of interest to them, they will respond with details about the ad and a bid offer in CPM. If not, a null bid response object will be sent. 2a. 2b. The ad exchange processes all the bid responses for the given bid request and declares the winner: the highest bid that fits all the requested parameters. The winning ad will be delivered and shown on the newspaper's article page within 100 milliseconds from the moment the use's browser accessed that page. Data about user behavior and post-click activity are usually collected by different systems for assessing campaigns and retargeting users.

The automated process is made possible by the work of the protocol and the set of digital objects that the protocol requires. The bid request and bid response objects talk to each other via a set of embedded objects and pre-set parameters that allow the exchange to happen. One of the most important objects of the RTB protocol is the ad impression object. Ad impressions are digital objects that have been set up to represent the likelihood of a single user to view an ad. An ad impression is defined as "the moment (a window of opportunity) between the fetching of an ad from its source as the result of the user's browser activity and the delivery of the ad" [19]. Ad impression objects are encapsulated in the bid request object (Fig. 1: 1) that needs to contain at least one ad impression object to trigger the exchange. The ad impression object is at the same time the good being exchanged and the rules of the exchange (its attributes or parameters). It contains different attributes that refer to: type of ad (banner, 
video, native) subordinated to the impression object, size, price such as bid floor, if any, modality of exchange, such as for instance if the object is exchanged through private marketplace (PMP), the expected time lag between bid winning and effective ad delivery (exp) and an array of metrics.

AdX, in particular, supplies three metrics of the ad impression object: viewability, click-through rate and video completion rate. Viewability is an estimate of the likelihood that the ad slot will be viewable by the end user, based on a range of indicators. It is expressed as percentage and calculated by taking into account historical data on user behavior and ad slot attribute data such as certain pixel and exposure thresholds, usually counting a minimum of $50 \%$ of the ad's pixel exposure for 1 or 2 continuous seconds [23, p.7], although this may vary across exchanges. Viewability refers to the likelihood or "opportunity to see". It does not therefore signify or stand for an actual viewing of the ad by the end user. It rather counts surrounding or environment factors of the ad slot such as visibility (if the ad is above or below the fold), a threshold exposure and a set of behavioral data on user (if available). These attributes can also be used as a selection and filtering criteria to find impressions and ad slots worth responding and bidding on. In this respect, viewability -an estimate of the likelihood or opportunity to see- becomes the criterion shaping a decision to bid in the pre-bidding phase.

\section{Discussion: conjectural Objects and the Management of Time}

The automation of real time bidding in the programmatic domain depends on a very complex IT infrastructure which is quickly growing both in scale and scope. The complexity arising from the automation of the core exchanges in an over-fragmented landscape of actors, business contexts, media and technologies is partially dealt with the establishment of a range of new objects. These include ad impressions, bid requests, action requirements and templates and, crucially as our study suggests, a range of metrics that lend facticity to these objects and monitor their movements along the virtual space of programmatic advertising $[10,17,20]$.

It is worth pointing out that these metrics act as soft industry or market standards. By this we mean that measures such as viewability in essence work as acceptable representations of the function and facticity of the entities or objects of programmatic advertising that -despite the structuring they are subject to by the real-time bidding protocol (RTB)- remain evasive. A good example that we have repeatedly touched upon is the fundamental entity of ad impression. What is an ad impression in fact? Ad impression has an institutional history yet it does not have a physical reality outside the advertising ecosystem.

As we have shown in the literature review section, ad impression has been the object of conflicts and highly contested definitions. A common definition of ad impression such as the "advertisement that [was] sent to an individual who had clicked on a site's page" [29] brought conflicts on issues of what is exactly delivered and whether this is actually viewed by the user. This is of particular relevance given that ad impressions are the building blocks of the CPM price structure, what marketers pay for. 
The advent of programmatic signaled yet another change to the definition of ad impressions that still remain highly contested. Ad impressions are now defined as the time lapse between a user's browser fetching a webpage with available ad slots and the effective display on the webpage of ads. This definition would suggest that every time a single browser fetches a webpage with at least one available ad slot a single ad impression is produced and exchanged. It is crucial to make clear that this change is of far reaching significance. Traditionally, impressions were quantified by ex post audience measurements, i.e. newspaper readership or television audience share constricted by panel measurements. Now, by contrast, they are sold ex ante, ahead of being effectively produced (i.e. the time lapse before ads are displayed) and therefore prior to user's attention being captured and measured.

Effectively, marketers bid on the likelihood that users see their ads. The metric of viewability is a way of fixing the unstable nature of ad impressions. Yet, these metrics are just industry conventions, the outcome of the negotiation of field participants with respect to how to manage the ambiguous nature of ad impressions and the intangible character of exchanges between actors and machines that on most essential respects are no more than data exchanges of future events. Viewability and click-through rates are the metrics intended to lend facticity to ad impressions. As it is often the case with metrics, because of the possibility of counting, viewability of impression appears as a fact rather than an object whose embedment in reality is variously problematic. These metrics are themselves hard to grasp. They are usually hidden away within complex multi-layered digital objects (viewability is an attribute of an ad impression object encapsulated in a bid-request object). They are estimates or likelihoods subjected to the limits, conventions and affordances of data tracking, recording and computing systems. They are often the result of complex industry negotiations [7] and they are ill-tolerated standards which have lost both their link with reality and their credibility within the industry.

Drawing on Elena Esposito, we call the entities constructed by these metrics conjectural objects $[11,12]$. The term indicates that such objects do not exist as independent givens but only as relational constructs involved in the representation of yetto-be objects. They are relational to the degree that they exist in a matrix of connections to other objects and actions and forward looking in the sense of seeking to regulate future occurrences. Conjectural entities are registered in other domains. They can be interest-rate shaping objects such as the LIBOR [13, 21, 22], ratios, indexes and soft standards such as measures that help structure actors' expectations by consolidating a practice that develops around their exchanges. In the case of financialderivatives, for instance, such objects were developed to solve "the most pressing issue which was standardizing the underlying asset to an extent sufficient for claims on it to be tradable without reference to any specific physical entities" [22, p. 368].

A similar issue, we suggest, is present in the programmatic advertising domain, where the exchange is based on non-physical, yet-to-be objects such as ad impressions whose future realization is contingent on the interplay with other objects. As of today, one of the industry leading bodies, the IAB (Interactive Advertising Bureau) is pushing for the adoption of the "viewable impression" object. Viewable impressions are ad impressions that match "certain pixel and time exposure thresholds (minimum 
$50 \%$ of the ad's pixel for 1 or 2 continuous seconds for display and video respectively)" [23, p. 7]. As the Media Rating Council (MRC) digital audience-based measurement standards document continues: "viewable impressions are now the minimum required unit for digital audience-based measurement including digital and crossmedia reach, frequency and GRP [Gross Rating Point]" [23, p. 7].

The excerpt is important because it attests to the shift in modes of measuring attention (and audiences) which now relies, rather heavily, on the affordances of the automated exchange. Standards such as viewability and pixels cannot measure user (actual) response. They can only count the time an ad can be visible on a page and the position the ad has on the screen (machine-to-machine). These metrics can be coupled with click measurement and other data as they are acquired from Data Management Platforms to calculate the likelihood of an ad being viewed. Audience measurement (reach, frequency and GRP) thus becomes the aggregation and computation of viewable impressions -while the ad impression measurability lends facticity to user attention.

It is worth to point out that there is no human agency involved here as these measurements and standards develop in highly automated exchanges, whereby machines talk to other machines in quick and massive interactions of data flows. Humans are of course the initiators of the process both in the form of end users whose clicking behavior triggers the web browser's request of an ad and, certainly, through the many actions and decisions of key industry actors that have been essential in setting up this hyper-complex ecosystem. However, the programmatic exchange of data, objects and measures which make today's digital advertising ecosystem -including the "useraudience" concept- is designed to operate without any further involvement of humanagents (on either side). As we have just seen, viewability is designed to measure the involvement of human users by substituting it with machine-readable metrics such as time and exposure of an ad on screen, plus history of clicks or profiles compiled and sold by third-party, data analytics, and platforms.

Conjectural objects arise out of the mutual expectations of the actors involved in the exchange and the infrastructure underlying the exchange, and act as reliable terms of reference for the actors involved. They are conjectural because they are involved in lending facticity to a promise that has yet to happen. It is crucial to clarify that the programmatic is neither involved in selling ads nor ad impressions (what would these be anyway?) The ecosystem rather trades the computable likelihood of an ad to be seen by a user, or as the industry calls it an "opportunity to see". This, we believe, makes them a different kind of objects that seem particularly germane to study, given their performative role in producing what is measured and exchanged, and how it is exchanged in the field of programmatic advertising. Effectively, here performativity assumes another dimension as it signifies the involvement of machines in the objects being measured which in the case of advertisement are audience and user attention. Even though IS as a field has only intermittently and indirectly dealt with these issues $[1,27]$, the debate on the facticity of concepts such as "audience" and "attention" has a long history in sociology and media studies, as pointed out in the introduction. Yet, the current developments that coincide with the diffusion of programmatic advertising aggravate the difficulties of establishing a field whose commercial relations are con- 
tingent on estimates and likelihoods of future occurrences realized out of the interactions of networks of intangible objects. As our empirical study illustrates, these difficulties are addressed not with a turn to reality but with a further leap into the conjectures of a probable world whose articulation (and calculation) requires the further separation of the entire ecosystem from real life events and the capabilities of human actors.

The advent of a data-driven rationalization paradigm in audience and attention measurement and the rush toward measurability has over time changed the objects observed. Traditional audience and attention mapping techniques measured content consumption with panels and adopted metrics and ranks on publisher outlets and reputations, audience habits and so forth. In the new paradigm we have presented here, publishers and their outlets have lost ground and power because marketers understood that the "click" was giving them the possibility of following user across contexts independently from publishers. The passage shifted the attention from the publisher to the user, making content outlets (webpages) less important. The machinery of ad networks relegated web publishers further into the background as on ad networks' auctions marketers actually bid on impression (user attention) without seeing where their ads will go. Ad networks made publishers literally invisible, contributing to their loss of power in the industry.

The advent of ad exchanges, we contend, has brought yet another important shift as it changed the modality in which audience measurement takes place and thus the object measured (audience). As seen, within the programmatic domain human-users loose importance as actors and as observable objects. In fact, their role is marginal for the effective completion of the exchange process. To work effectively on such a large scale, programmatic needs to replace human involvement with machines: software, platforms, and objects. In a sense, viewable impressions are purposely designed to replace other indicators to measure audience response with machine-readable ones. The unintended consequence of setting up such a complex data exchange and communication ecosystem that replaces human-machines assemblages with nearly closed circuits of machines-to-machine communication is the rise of what we conceive as path-dependent megastructures, massive automated infrastructures where each layer generates integral accidents that are solved by adding other layers $[8,10]$.

\section{Final remarks}

Our empirical study suggests that the field of programmatic advertising carries the standard problems of attention capture and audience measurement into an entirely new stage by setting up a hyper-complex machinery of data tracking tools, semiautomated platforms, bidding algorithms and protocols. This technological complex and the practices and interests to which it is related establish a new institutional context in which attention is defined and tracked in new ways that further technicalize the perception and measurement of it and considerably automate the ways it is being traded and monetized. The changes we document have key attributes of a self-referential, or path-dependent, process whereby each step taken draws on, reinforces, and devel- 
ops what is already in place [7, 16]. The outcome of this process is the creation of larger enclaves of automated sequences that considerably tweak and replace older practices with machine-to-machine data exchanges and which, in the case of conjectural objects, trade the future for the present [11].

By linking audience measurement to the technological affordances of automated exchange in massive platform ecosystems we contribute to the study of automation and of its consequences. Our findings clearly indicate a drifting away of measurement techniques from user behavior data to contextual data, something that allows for a faster and smoother exchange as it mostly relies on the mutual programming of digital objects. Furthermore, what we observe in the field of digital advertising has parallels to other industries which are becoming increasingly dominated by the reciprocal programming of conjectural objects such as finance and, increasingly, the digital travel industry sector. Programmatic advertising is then part and parcel of broader changes in which human exchanges are increasingly infiltrated and structured by the technological dynamics and the economic interests they serve.

\section{$7 \quad$ References}

1. Aaltonen, A. and Tempini, N., 2014. Everything counts in large amounts: A critical realist case study on data-based production. Journal of Information Technology, 29(1), pp. 97110.

2. Alaimo, C. and Kallinikos, J., 2017. Computing the everyday: Social media as data platforms. The Information Society, 33(4), pp. 175-191.

3. Anderson, C. 2007. The Long Tail: How Endless Choice is Creating Unlimited Demand. New York: Random House.

4. Ang, I., 2006. Desperately seeking the audience. London: Routledge.

5. Bermejo, F. 2007. The Internet Audience: Constitution and Measurement. New York: Lang

6. Bermejo, F. 2009. Audience Manufacture in historical Perspective: From Broadcasting to Google. New Media and Society. 11 (1). pp. 133-154

7. Bowker, G. C. and Star, S. L. 1999. Sorting things out: Classification and its consequences. Cambridge MA: MIT press.

8. Bratton, B.H., 2016. The stack: On software and sovereignty. Cambridge MA: MIT press.

9. Carmi, E. 2017. Regulating behaviours on the European Union internet, the case of spam versus cookies, International Review of Law, Computers \& Technology

10. Ciborra, C. 2006. Imbrication of representations: Risk and digital technologies. Journal of Management Studies, 43(6), pp. 1339-1356

11. Esposito, E. 2008. Probabilità improbabili. La realtà della finzione nella società moderna. Roma: Meltemi Editore.

12. Esposito, E. 2013. The structures of uncertainty: Performativity and unpredictability in economic operations. Economy and Society, 42(1), pp. 102-129

13. Ettema, J.S. and Whitney, D.C., 1994. Audiencemaking: How the media create the audience. London: SAGE

14. Galloway, A.R., 2004. Protocol: How control exists after decentralization. Cambridge MA: MIT press.

15. Google for Developers, 2018 Real-Time Bidding Protocol, Online at: https://developers.google.com/ad-exchange/rtb/openrtb-guide 
16. Hanseth, O. 2000. The economics of standards. In C. Ciborra (Ed.), From control to drift: The dynamics of corporate information infrastructures, pp. 56-70, Oxford: Oxford University Press.

17. Hanseth, O. and Lyyttinen, K. 2010. Design theory for dynamic complexity in information infrastructures: the case of building internet. Journal of information technology, 25(1), pp. $1-19$.

18. IAB (Interactive Advertising Bureau), 2016. Open RTB Protocol Specification (API specification v2.5, 2016): S7. Online at: http://www.iab.com/wpcontent/uploads/2016/03/OpenRTB-API-Specification-Version-2-5-FINAL.pdf

19. IAB (Interactive Advertising Bureau), 2004, Interactive Audience Measurement and Advertising Campaign Reporting and Audit Guidelines, Online at: https://www.iab.com/wpcontent/uploads/2014/10/Ad-Impression-Measurement-guideline-Global.pdf

20. Kallinikos, J. 2005. The order of technology: Complexity and control in a connected world. Information and Organization, 15, pp. 185-202.

21. MacKenzie, D. 2006. An Engine, Not a Camera. How Financial Models Shape Markets. Cambridge MA: MIT Press.

22. MacKenzie, D. 2007. The material production of virtuality: Innovation, cultural geography and facticity in derivatives markets. Economy and Society, 36(3), pp. 355-37

23. MRC (Media Rating Council) and IAB (Interactive Advertising Bureau), 2017. Digital Audience-Based Measurement Standards v5.1 Public Comment Draft. Online at: http://mediaratingcouncil.org/MRC\%20Digital\%20Audience-

Based\%20Public\%20Comment\%20Draft.pdf

24. Napoli, P.M., 2011. Audience evolution: New technologies and the transformation of media audiences. New York: Columbia University Press.

25. Napoli, P.M., 2012. Audience economics: Media institutions and the audience marketplace. New York: Columbia University Press.

26. Schwartz, J. 2001. Giving Web a Memory Cost its Users Privacy, The New York Times, Sep. 4, 2001, Online at: https://www.nytimes.com/2001/09/04/business/giving-web-amemory-cost-its-users-privacy.html

27. Scott, S.V. and Orlikowski, W.J. 2014. Entanglements in practice: Performing anonymity through social media. MIS Quarterly, 38 (3), pp. 873-893.

28. Taneja, H. and Mamoria, U., 2012. Measuring media use across platforms: Evolving audience information systems. International Journal on Media Management, 14(2), pp. 121140.

29. Turow, J., 2011. The daily you: How the new advertising industry is defining your identity and your worth. Yale University Press.

30. Webster, J.G., 2014. The marketplace of attention: How audiences take shape in a digital age. Cambridge MA: MIT Press.

31. $\mathrm{Wu}, \mathrm{T} ., 2017$. The attention merchants: The epic scramble to get inside our heads. Vintage.

32. Yin, R. 2013. Case Study Research Design: Design and methods. London: Sage.

33. Yoo, Y., Henfridsson, O., \& Lyytinen, K. 2010. Research commentary--the new organizing logic of digital innovation: An agenda for information systems research. Information Systems Research, 21(4), pp. 724-735. 